\title{
Design of photoelectric pulse device based on Bluetooth
}

$$
\text { LIU Qiang }{ }^{1, a} \text {, LOU Liang-hua }{ }^{1, b}, \text { ZHANG Tao }{ }^{2, c} \text {, and ZHU Yong-yun }{ }^{2, c}
$$

${ }^{1}$ Big data Key Laboratory of the Ministry of Public Security, Zhejiang Police College, Hangzhou 310053, China

\author{
${ }^{2}$ School of Instrument Science and Engineering, Southeast University, Nanjing 210096, China

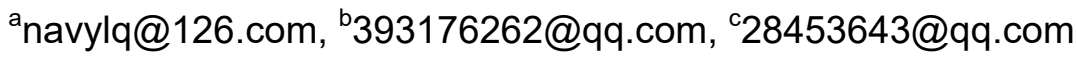

\begin{abstract}
Keywords: Photoelectric sensor, pulse detection, micro controller, Bluetooth data transmission.
\end{abstract}
\begin{abstract}
The principle of using photoelectric sensor to detect the pulse waveform is introduced. A low cost photoelectric pulse detection device is designed, which can improve the pulse detection circuit. In addition, the pulse signal waveform display $\mathrm{PC}$ is designed by using $\mathrm{C \#}$ language, and the pulse waveform signal can be transmitted by Bluetooth wireless transmission. The detection device is mainly composed of a signal acquisition circuit, signal conditioning circuit, microcontroller STM32 signal processing, Bluetooth data transmission, PC waveform display components, principle and analysis process of each part of the detecting device are introduced in detail. From the experimental results, the detection method of the device is simple, high sensitivity, and greatly reduce the cost of pulse signal detection.
\end{abstract}

\section{Introduction}

Pulse signal is caused when the heart beats in the blood vessels to the result of the spread of function, reflects the comprehensive information of the human physiological system dynamics. It is an important physiological indexes of clinical tests which can be extracted from the pulse signal important related parameters and provide important information for medical research. In clinical test, we can gets a lot of diagnostic value by pulse wave information. Because the change of physical properties of the pulse signal before clinical symptoms usually appear in the attack, so you can adopt to these practical information to analyze the health of the human body organs and forecast the change trend of the structure and function of various organs.

Common methods of measuring heart rate are heart electric detection, heart sound detection and photoelectric detection. The heart electric detection results are accurate and the heartbeat signal contains a variety of effective parameters, also have a high demand for electrode materials; Heart sound detection can obtain the most original heartbeat waveform data, but the heart sound detection has strong signal interference, and effective signal acquisition is difficult, and the signal noise is relatively light; Photoelectric detection is easy to use, practical, and its waveform effect is good. It has high sensitivity and costs less. So this design uses photoelectric detection method to detect the human pulse waveform signal.

\section{Working principle}

Hemoglobin $\left(\mathrm{H}_{\mathrm{b}}\right)$ has different absorption spectrum characteristics under different oxygen conditions, so in the blood of the human body, the hemoglobin $\left(\mathrm{O}_{2} \mathrm{H}_{\mathrm{b}}\right)$ and the hemoglobin $\left(\mathrm{H}_{\mathrm{b}}\right)$ which are not oxygenated have different absorption coefficients for different wavelengths of light. In the infrared region, the absorption coefficient of $\mathrm{Hb}$ is less than $\mathrm{O}_{2} \mathrm{H}_{\mathrm{b}}$. When the oxygen rich blood comes, the infrared light will be absorbed more, causes the signal to transmit to the photoelectric receiving tube to weaken, and vice versa. According to this principle, the pulse detection device can be designed, and the schematic diagram is shown in Fig. 1.

Detection mainly includes transmitting and receiving. Light emission control infrared emitting tube emits infrared light signal. The human heart will cause changes in the fingertip blood flow. When the infrared emission circuit from the infrared light onto a finger, the amount of transmitted 
light of the infrared receiving tube receives will be changed. At the same time, the optical current in the receiving circuit is changed. Therefore, the change of heart beating caused by fingertip blood flow change the size of the received light intensity by photoelectric pulse sensor. And through the optical measurement circuit will receive the change of light intensity change to the change of the voltage value. The pulse signal is filtered by the signal conditioning circuit and the output voltage is amplified to the appropriate size. Then by the acquisition AD collection and transmission of the voltage signal to the microcontroller, through STM32 microcontroller can get pulse signal effectively. Finally, the pulse signal is transmitted to the host computer through the Bluetooth wireless module.

\section{Hardware design}

Light measurement circuit. The optical emission circuit of the sensor is used as an infrared transmitting tube. When the electric current of the infrared transmitting tube is enough to make the tube work, the infrared light (invisible light) can be emitted. The tube voltage drop of infrared LED is about $1.4 \mathrm{~V}$, and the operating current is $3-20 \mathrm{~mA}$. In this design, the optical emission circuit is powered by more than $5 \mathrm{~V}$ power supply. Current limiting resistance of $120 \Omega$ is in the loop.
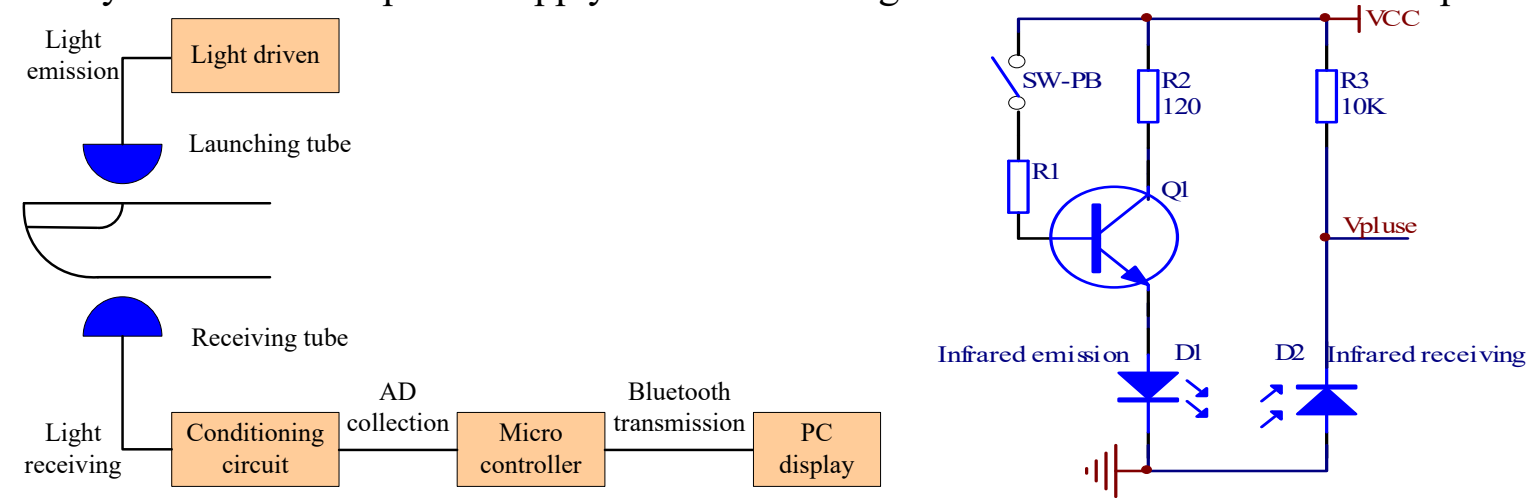

Fig. 1 Principle of pulse detection

Fig. 2 Principle of light measurement circuit

The photosensitive diode light intensity can be converted into voltage or current information. It has good linearity and high sensitivity, can be used as a photoelectric pulse detection device of light receiving device. Therefore, the design of the light receiving device to select a photosensitive diode, the same in the light receiving circuit also need to add the partial pressure limiting resistance. The design of the optical receiving circuit using $5 \mathrm{~V}$ power supply and the limit current resistance is 10 $\mathrm{k} \Omega$.

In the Fig. 2, When SW-PB is switched on, the transistor Q1 turns on. At this point, the infrared light emitting diode works, the photosensitive diode receives the light signal, the photoelectric rheology becomes larger, the voltage drop across the resistance R3 becomes larger, thus the output voltage of the Vpluse terminal is reduced. When the SW-PB is disconnected, the transistor Q1 cut-off, at this time the infrared light emitting diode does not work, the photosensitive diode can not receive the light signal, showing a high resistance characteristics, the output voltage of the Vpluse terminal at this time is higher. When SW-PB switched on, Finger is placed in the infrared tube, finger blood flow will continue to change with the heart. In this case, the light intensity received by the light sensitive diode is also changed, which can cause the change of the output Vpluse terminal level to obtain the pulse signal.

Signal conditioning circuit. As shown in Fig. 3, The signal conditioning circuit of the pulse detection device is composed of a front stage amplifying filter, a back stage amplifying filter, a voltage follower and a Schmidt trigger. The signal conditioning circuit output signal access AD microcontroller STM32 acquisition pin. In the course of the heartbeat, the blood flow of the fingertip changes, the output voltage of the signal conditioning circuit will change accordingly, so the microcontroller can get different voltage values. After digital filtering in the micro controller, the signal is calculated to determine the pulse rate per minute, then it will display the pulse wave signal is sent to the host computer via Bluetooth wireless module. Based on the aforementioned hardware design, the design of the hardware system, as shown in Fig. 4 and Fig. 5. 


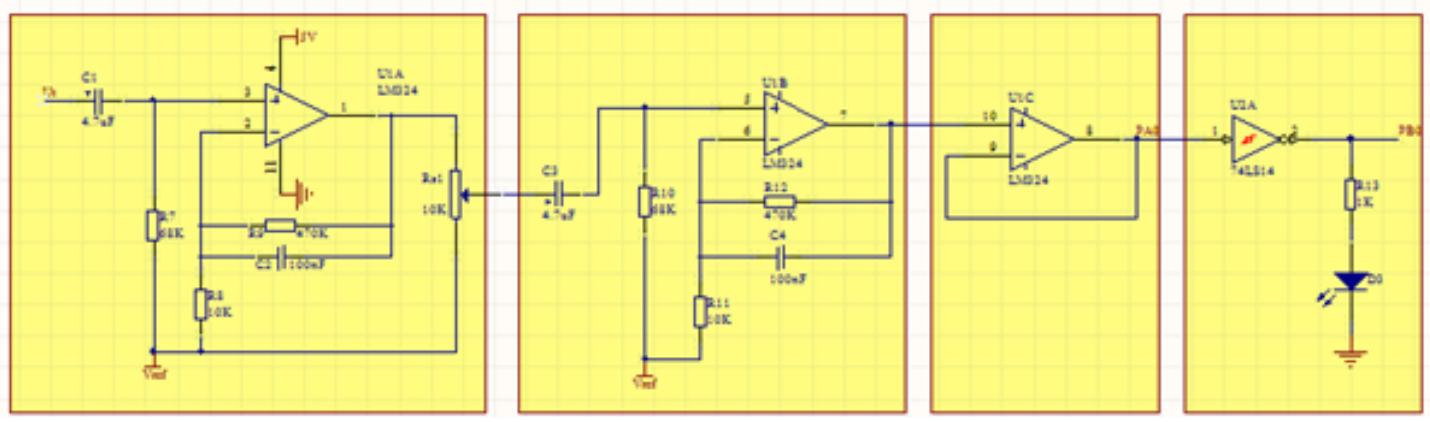

Fig. 3 Signal conditioning circuit

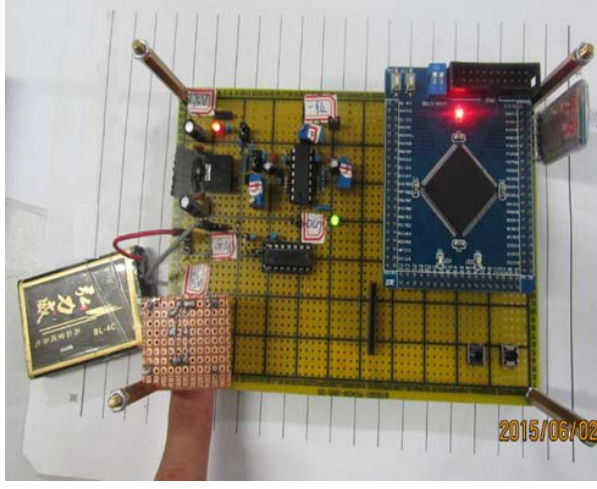

Fig. 4 The circuit board

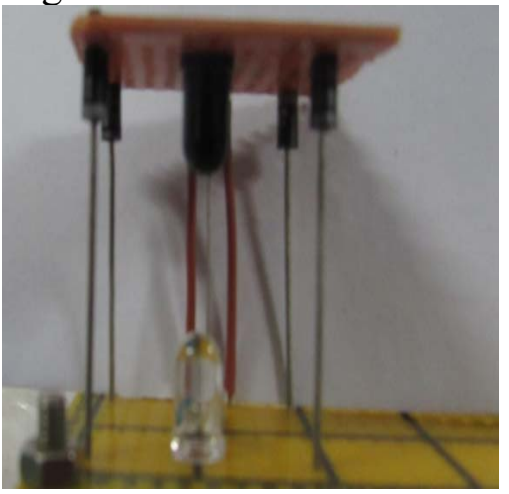

Fig. 5 The pulse acquisition sensor

Pre amplifier filter circuit. The pulse detection device for signal conditioning circuit pre amplifying circuit adopts a voltage controlled voltage source second order active low-pass filter circuit. When the change of the output of the optical receiving circuit caused by the change of the blood flow of the finger tip is changed, the pre amplifier filter circuit which is composed of an operational amplifier is connected with the output terminal voltage. Because the circuit is a low pass filter circuit, so it can not only remove the DC signal, but also filter out the high frequency interference In addition, the circuit uses a single power supply, in the amplifier circuit to raise a level Vref, so that the entire cycle of the pulse signal can be amplified processing.

By analyzing the circuit can be obtained, the output voltage expression of the circuit is

$$
V_{o(s)}=V_{r e f(s)}+V_{i(s)}\left(1+\frac{R_{2}}{R_{3}\left(1+R_{2} C_{2} s\right)}\right)
$$

According to the formula $A_{v}=1+R_{9} / R_{8}$, we choose two resistances $\left(R_{9}=470 \mathrm{k} \Omega, R_{8}=10 \mathrm{k} \Omega\right)$. At the same time in order to eliminate the bias voltage, in the negative input and the common end of access to resistance $R_{7}$. The resistance of $R_{8}$ and $R_{9}$ are both $9.8 \mathrm{k} \Omega$, the two resistances are parallel.

According to the formula $\mathrm{f}=1 /\left(2 \pi \mathrm{R}_{9} \mathrm{C}_{2}\right)$, Low pass cutoff frequency is set at $3 \mathrm{~Hz}$. Because the resistance of $R_{9}$ is $4.7 \mathrm{k} \Omega$, so we choose the capacitance value of $C_{2}$ is $100 \mathrm{nF}$. Low pass cutoff frequency is approximately $3.39 \mathrm{~Hz}$. Pass band as shown in Fig. 6, which is $0-8.99 \mathrm{~Hz}$ 。

Voltage follower and Schmidt trigger. The voltage follower shown in Fig. 3, which circuit input resistance tends to infinity, the output resistance tends to be infinitely small, can play a buffer, isolation and improve the capacity of load. After the pulse signal passes through the sensor as well as the amplification filter circuit, the voltage signal which is obtained is the simulation quantity which continuously changes. In order to count the pulse signal, we need to get the square wave signal through the voltage comparator circuit. However, the single threshold voltage comparator output 
waveform contains a lot of small clutter. So this design uses Schmidt trigger to get a more standard square wave signal.

\section{Software design}

Lower machine software design. Fig. 7 is the process of the pulse detector software. The function key through the external interrupt to judge whether the button is pressed or not. It has high sensitivity and accurate judgment. After the signal conditioning circuit, the pulse signal is processed to the appropriate size. The information collected by the microcontroller can be converted to digital information by the sampling voltage value. After the digital filtering, you can calculate the number of pulses. Finally, the pulse signal is transmitted to the host computer by Bluetooth wireless module. At the same time, the pulse waveform data is displayed in the upper computer.
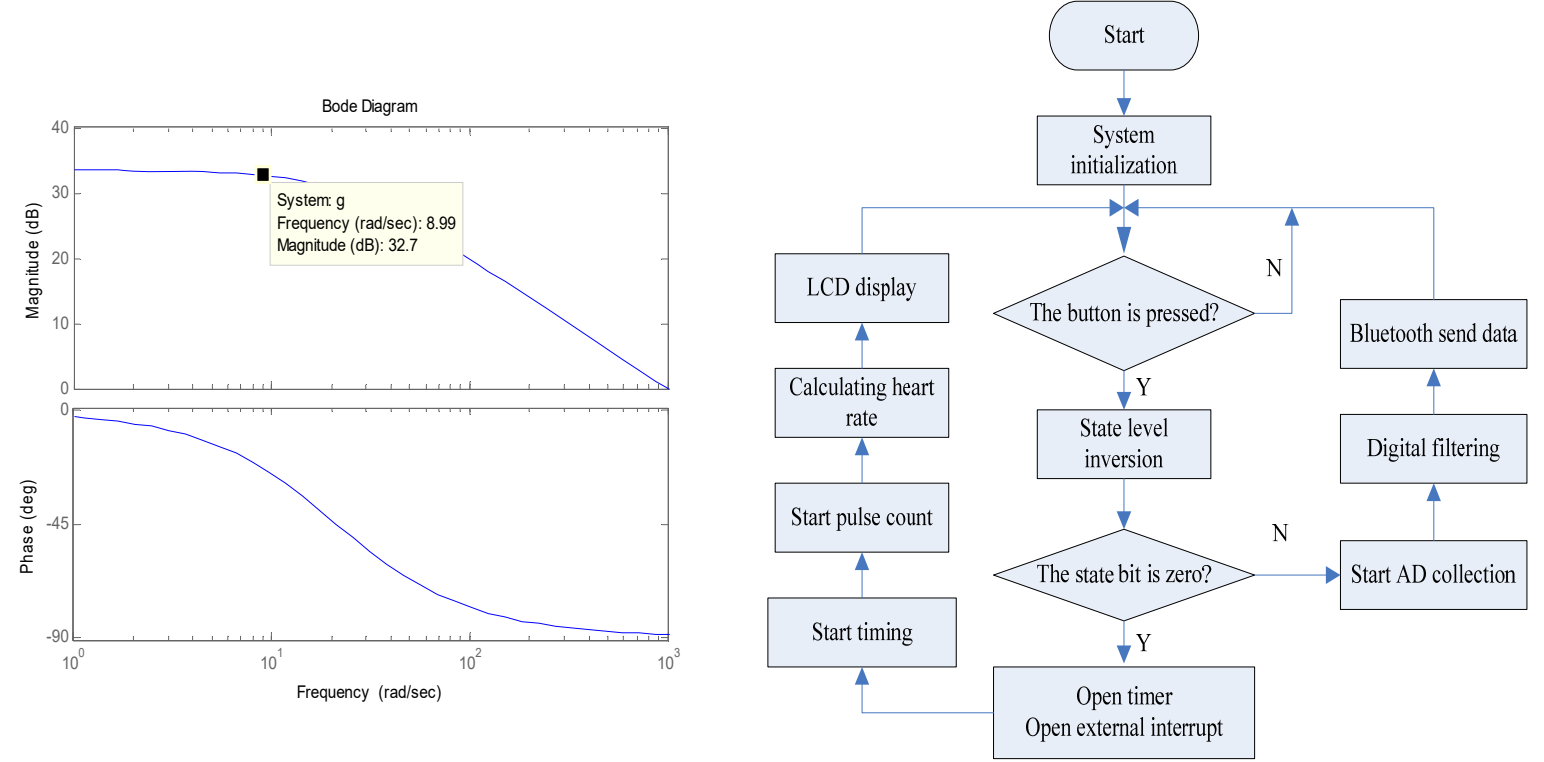

Fig. 6 Pre amplifier filter circuit Potter Fig. 7 The process of the pulse detector software

Upper monitor software design. The upper monitor uses $\mathrm{C \#}$ language to carry on the design. The design effect of the front panel is shown in Fig. 8. The front panel area design mainly includes the waveform display area, the serial port parameter setting area as well as the key control area. Part of the program design includes window initialization (For each area window size, serial port parameters, data storage initialization), data receiving and processing (Receive and process data), waveform display (Display of axis and waveform of waveform display area) and control key processing (Waveform amplification, waveform reduction, waveform preservation button).

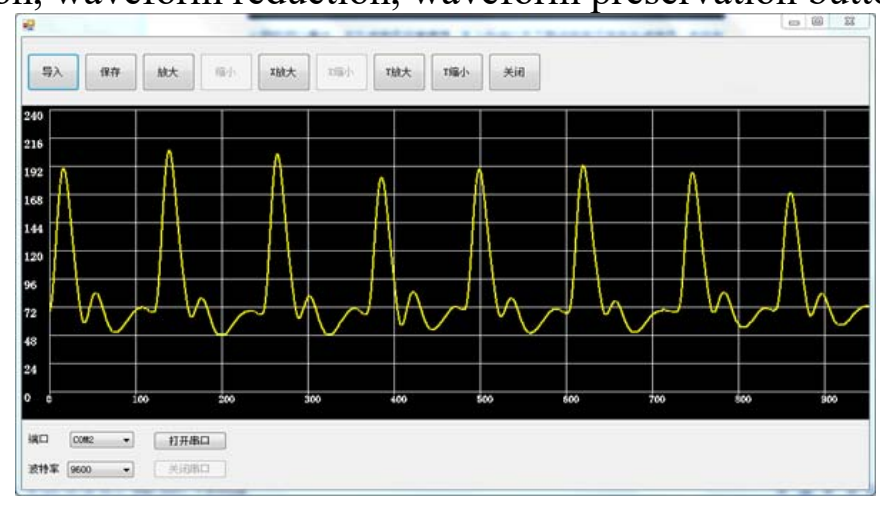

Fig. 8 The upper monitor design Interface

\section{Experimental result}

In the experiment, the signal conditioning circuit output signal is connected to the oscilloscope to observe the observation effect as shown in Fig. 9. 


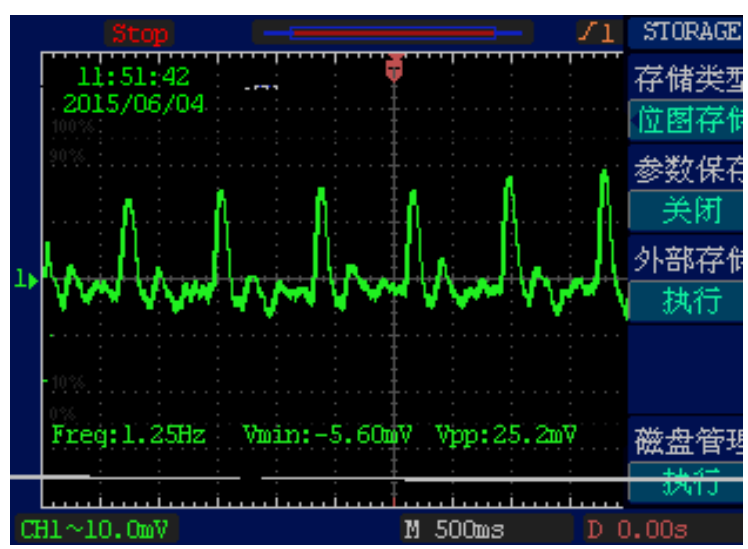

Fig. 9 Display of pulse waveform acquisition of the original signal waveform output signal

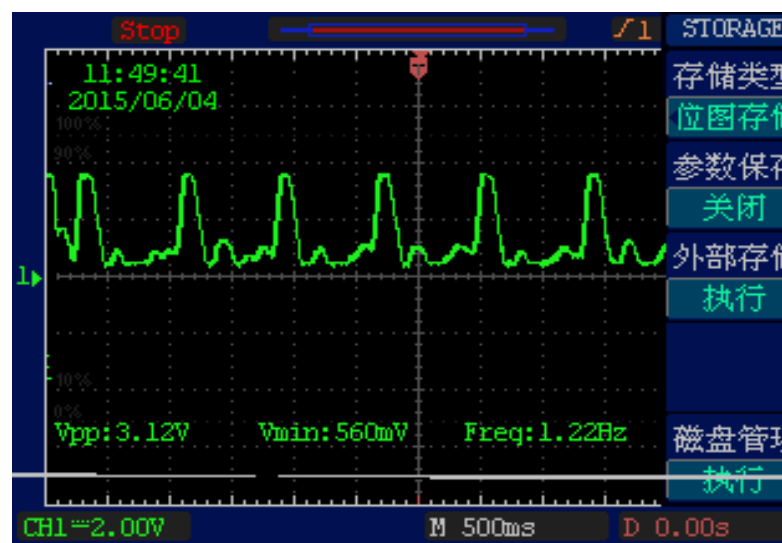

Fig. 10 Display of pulse

Fig. 9 shows the original pulse waveform signal obtained by the sensor signal acquisition circuit which Peak value is about $25 \mathrm{mV}$. Fig. 10 shows the signal conditioning circuit for the pulse of the original signal processing after the waveform signal which Peak value is about 3.1 V. Then the signal is transmitted through the Bluetooth module, and the pulse waveform signal is displayed in the upper monitor. The effect of waveform is shown in the Fig. 8.

\section{Summary}

This design uses STM32F103ZET6 microcontroller as the main control chip, using Bluetooth technology as the way of data transmission, using photoelectric pulse sensor, combined with a set of design scheme of pulse signal detection instrument of upper monitor. The key to the design of the device lies in the design of the photoelectric sensor and the processing of the pulse signal. From the test results, the device to detect the pulse waveform is clear, accurate data, with a high practical value. The device has the advantages of simple production method, low cost, convenient use and reliability. It can realize the pulse wave signal detection with good function.

\section{Acknowledgements}

This work was financially supported by the NSFC-Zhejiang Joint Fund for the Integration of Industrialization and Informatization(U1509219), National Undergraduate Training Programs for Innovation and Entrepreneurship National Project(201411483001), Students Science Technology Innovation Activities and Xinmiao Talents Project of Zhejiang Province(2015R432011), and Zhejiang Police College Research Project (20140104).

\section{References}

[1] X.P. Zhou: Sensor and detection technology (Tsinghua University press, Beijing 2010).

[2] G.H. Kang: Electronic technology foundation (Higher Education Press, Beijing 2005).

[3] S. Yan: Digital electronic technology foundation (Higher Education Press, Beijing 2005).

[4] G. Peng: Application of STM32 series embedded microcontroller based on Cortex-M3 (Publishing of Electronics Press, Beijing 2011).

[5] J.H. Wang, and A.H. Zhang. Journal of Lanzhou University of Technology Vol. 35 No. 4(2009), p. $78-83$. 